\title{
Philosophiques
}

\section{Le concept de liberté chez Herbert Marcuse}

\section{Jean-Claude Clavet}

Volume 13, numéro 2, automne 1986

URI : https://id.erudit.org/iderudit/203317ar

DOI : https://doi.org/10.7202/203317ar

Aller au sommaire du numéro

Éditeur(s)

Société de philosophie du Québec

ISSN

0316-2923 (imprimé)

1492-1391 (numérique)

Découvrir la revue

Citer cet article

Clavet, J.-C. (1986). Le concept de liberté chez Herbert Marcuse. Philosophiques, 13(2), 209-235. https://doi.org/10.7202/203317ar

\section{Résumé de l'article}

Parce que les sociétés industrielles avancées sont générées par l'idéologie et la fécondent, elles sécrètent une liberté malheureuse. La phénoménologie et la critique marcusiennes des expressions dominantes de ces sociétés s'appliquent à le montrer. Toutefois, les exigences mêmes d'une pensée négative obligent à circonscrire les normes de transformation nécessaire et à prescrire ce qui doit être.

Cette réflexion se veut un exposé de l'approche de la liberté proposée par la " théorie critique ». d'utilisation que vous pouvez consulter en ligne.

https://apropos.erudit.org/fr/usagers/politique-dutilisation/ 
PHILOSOPHIQUES, Vol. XIII, Numéro 2, Automne 1986

\title{
ARTICLES
}

\section{LE CONCEPT DE LIBERTÉ CHEZ HERBERT MARCUSE}

\author{
par Jean-Claude Clavet
}

RÉSUME. Parce que les sociétés industrielles avancées sont générées par l'idéologie et la fécondent, elles sécrètent une liberté malheureuse. La phénoménologie et la critique marcusiennes des expressions dominantes de ces sociétés s'appliquent à le montrer. Toutefois, les exigences mêmes d'une pensée négative obligent à circonscrire les normes de transformation nécessaire et à prescrire ce qui doit être.

Cette réflexion se veut un exposé de l'approche de la liberté proposée par la «théorie critique».

ABSTRACT. Because advanced industrial society was conceived by and nurtures ideology, it yields unhappy freedom. Indeed, the purpose of Marcuse's phenomenology and critique of the dominating features of these societies is to demonstrate this view. Principles of negative thinking, however, require that the norms of a necessary transformation of these societies be defined, and to prescribe what must be done.

This paper presents the approach to freedom as suggested by critical theory.

Deux ans après l'immixtion des troupes américaines dans la guerre du Vietnam, Herbert Marcuse soutient, dans un texte majeur de son itinéraire philosophique, que:

«En tant que processus historique, le processus dialectique implique la prise de conscience: la reconnaissance et l'appréhension des possibilités de libération. Elle implique ainsi la liberté. Or, les exigences et les intérêts ont déterminé la conscience à tel point qu'elle n'est pas libre; la société est irrationnelle à un point tel que la conscience ne peut 
atteindre à la liberté de la rationalité supérieure qu'en engageant la liberté contre la société établie» ${ }^{1}$.

Cet extrait introductif campe d'emblée la problématique de la liberté chez le penseur de l'École de Francfort. Non seulement précise-t-il les références méthodologiques de l'auteur mais il situe de façon péremptoire la thèse marcusienne de l'absence de liberté dans les sociétés industrielles avancées et annonce les conditions nécessaires à l'institution d'une société libre.

Le texte marcusien apparaît habité d'une réflexion sur la liberté. Bien avant l'instauration du couple explicatif Marx-Freud retrouvé dans Éros et Civilisation, Marcuse affirme ${ }^{2}$ que l'une des tâches essentielles de la pensée philosophique est constituée du questionnement de la notion de liberté dans les modèles de représentation dominants des sociétés politiques contemporaines. Ce devrait être là la conséquence même d'une réflexion entendue comme "théorie sociale» ${ }^{3}$ s'appliquant à inspecter les manifestations signifiantes de la réalité historico-sociale.

L'objet de cet exposé semble dès lors fixé : il s'agit de discuter de la thèse marcusienne de la liberté en se préoccupant de trois questions directrices: (1) quelle est la signification du projet philosophique pour Marcuse? (2) pourquoi Marcuse peut-il affirmer l'absence de liberté dans les sociétés industrielles avancées? et (3) quelles sont les conditions immanentes à la réalisation et à l'exercice de la liberté?

\section{LE PROJET PHILOSOPHIQUE MARCUSIEN}

La «théorie critique» trouve sa pleine signification par la recherche de l'attribution de son lieu épistémique dans la dynamique des savoirs. Elle se définit en ce sens tout aussi bien négativement que positivement. D'abord négativement en ce qu'elle refuse certaines qualités dévolues à d'autres discours. Ensuite positivement parce qu'elle sait préciser la forme de

1. L'bomme unidimensionnel, p. 246.

2. Voir notamment «Sur la philosophie concrète», pp. 150-151, «Autorité et famille» et Raison et Révolution, pp. 199-203.

3. Raison et Révolution, pp. 301 et subs. et L'bomme unidimensionnel, p. 143: « Pour la théorie sociale, reconnaître les faits, c'est les critiquer ». 
procès qu'elle favorise et qu'elle sait dégager les objectifs qui l'animent.

L'École de Francfort prétend que le discours théorique entendu au sens strict peut être classé sous trois catégories. ${ }^{4} \mathrm{Il}$ peut être dit de type «empirico-analytique», comme celui des sciences recourant au paradigme expérimental et postulant un rapport de réciprocité nécessaire entre le connu et le mesurable et la vérité et le vérifiable, et il a un intérêt pratique évident se traduisant par la complexification de l'appareillage technologique. Ce modèle discursif dont l'audience apparaît presque totale dans le monde contemporain constitue l'héritage de la pensée qui s'est développée durant la Renaissance, avec particulièrement Francis Bacon. Ce savoir consolidé et renforcé durant la Grande Révolution Industrielle du $\mathrm{XIX}^{\mathrm{e}}$ siècle institue une relation dialectique fort étroite avec la technologie et l'économie. C'est pourquoi il est possible aujourd'hui de parler de complexe scientificotechnologique. Une épistémologie de ce premier type de discours identifié révèle de plus que la dimension empirique fonde la validité du connu. La soumission de toute analyse aux faits en établit la valeur. Ainsi, en quelque sorte, pour le discours empirico-analytique, l'être est ce qui se montre dans l'ordre du réel. Ce qui, par conséquent, caractérise le plus fondamentalement ce discours qui n'est que le type général de la presque totalité des sciences régionales, ce sont tout autant son objectivisme et son positivisme que son intérêt pratique. L'objectivisme ressort du fait que les sciences en question considèrent l'objet étudié comme une réalité structurée en soi, indépendamment de la conscience l'interrogeant. Elles apparaissent ainsi corrélativement positivistes par leur prétention à exclure le sujet de leur description et de leur analyse. Selon elles, leur discours ne fait que regarder la réalité dans toute sa positivité. Leur valeur se renforce finalement parce que toute leur connaissance se construit dans le souci de l'opérationnalisation. D'où l'impossibilité de dissociation de la science et de la technologie.

Ces constatations épistémologiques s'inscrivent fort bien dans la compréhension de l'originalité de l'École de Francfort et

4. Cette catégorisation est un effort de J. HABERMAS, La science et la technique comme idéologie, Denoël, Gonthier, Paris, 1973, p. 145. Marcuse y souscrit. 
permettront plus tard de saisir les objections prononcées par Marcuse à l'endroit du discours empirico-analytique. Pour l'instant, il suffit de reconnaître que c'est par rapport à celui-ci que l'École circonscrira son champ et son ordre épistémiques.

Le discours peut être aussi dit «historico-herméneutique». Cette seconde catégorie s'applique à une clarification des phénomènes historiques par leur interprétation afin d'en dégager les principes de l'action politique, économique et/ou sociale. Ce qui importe ici, c'est de se placer face à l'ordre qui se fait et l'interroger pour discerner ses justifications voilées ou explicites. Le travail «historico-herméneutique» se rattache à une préoccupation dominante qui concerne la praxis humaine incarnée. L'homme se fait à travers une multiplicité de choix sociaux qui doivent être saisis et compris dans leurs ramifications diverses. En effet, ce discours pose la réalité dans sa genèse en s'efforçant d'expliquer le statut et l'origine historico-sociale de sa représentation. Il demande «pourquoi en est-il ainsi?». Les raisons dégagées constituent des faits qui se présentent comme explicatifs du phénomène interrogé. Jamais les fondements ultimes intrinsèques à une anthropologie intégrale ne sont recherchés.

Ces deux premiers modes discursifs identifiés par l'École de Francfort privilégient essentiellement l'ordre descriptif. Les réalités s'imposent dans leur facticité et elles se révèlent en les décrivant.

L'École de Francfort distingue une dernière catégorie de savoir. Celui-ci est de type critique et a une fonction émancipatrice. Elle reconnaît que la philosophie participe de cette troisième catégorie. Il y a tout lieu de croire même que sa participation se veut exclusive. Dès que les sciences régionales radicalisent leur discours et se réclament d'une autre attitude devant l'ordre réel, elles se nient en tant que telles. D'ores et déjà, elles transcendent leur champ épistémique. Force est de reconnaître que peu ou pas de sciences régionales osent le faire.

L'acte du philosophe, selon Marcuse, s'inscrit dans sa première manifestation comme une phénoménologie de l'existence humaine, engagée sous les modalités de l'être dans l'événement collectif et historique. La lecture de l'homme dans sa concrétude le dévoile tout autant comme résultat que comme origine des 
rapports sociaux. Parce que l'homme appartient ontologiquement à l'histoire qui le fonde, l'examen phénoménologique doit analyser les déterminants de l'homme en révélant leurs complémentarités, leurs ambiguïtés, leurs corrélations et leurs significations. Plus simplement: la philosophie s'efforce d'abord, grâce au modèle dialectique, de produire une description de l'existence concrète incarnée dans l'histoire. En ce sens, la tâche de la philosophie concrète jusque-là, reste identique à celle des sciences régionales.

Toutefois, le travail phénoménologique contient dans son intention descriptive un pouvoir critique. Il garantit, en effet, une révélation des contradictions du continuum historico-social, lesquelles perpétuent son irrationalité. La philosophie concrète juge la nécessité du dépassement des contradictions de l'ordre existant en référant à un a priori éthique. Parce que les modèles de représentation d'une société et le vécu collectif doivent garantir la présence effective de valeurs spécifiques, la philosophie concrète dénonce et critique les formes politiques établies et dominantes. Le projet philosophique passe de ce fait d'un effort phénoménologique à un engagement éthique.

Engagement éthique en un double sens : d'abord, parce qu'il s'applique, grâce à ses références axiologiques, à évaluer et à nier l'histoire donnée. Dans les textes postérieurs à l'article «Sur la philosophie concrète» où il s'affirme comme théoricien critique, Marcuse reconnaît le caractère primordial de ce second moment en identifiant la philosophie à la "pensée négative». Engagement éthique aussi et surtout parce que la philosophie trouve son sens en se préoccupant de l'accomplissement de la norme dans la situation historique concrète.

Partie d'une dénotation de la pratique dominante et de sa négation nécessaire, la pensée philosophique retourne à la réalité en prescrivant un impératif catégorique désormais obligatoire au niveau historique. "Par l'universalité et la nécessité de ses concepts (...) la Raison peut prétendre dépasser la facticité de ce qui est vers la réalisation de ce qui doit être ${ }^{5}$. Ainsi définie, la philosophie est conçue comme un exercice où la dialectique opère dans sa double tension, ascendante et descendante: l'action

5. Raison et Révolution, p. 67. 
historique jugée par l'idée normative (ou axiologique) devient le censeur de celle-ci. Marcuse affirme sans équivoque le caractère prescriptif et par là éthique de la philosophie concrète lorsqu'il écrit :

"Conduire l'existence à la vérité, c'est changer "réellement" l'existence dans la concrétude, et non pas seulement changer (en surface) ses formes et ses structures effectives (...); c'est changer la manière d'exister elle-même qui est à la base de toutes ses formes» ${ }^{6}$.

La mission éthique du projet philosophique connaît sa mesure complète dans une pensée politique et dans une praxis publique. Toute altération des modes d'expression de l'existence suppose un bouleversement de la rationalité dominante dans les pays industriels avancés. Celle-ci renvoie tout autant aux systèmes de représentation de masse, au complexe scientifico-technologique qu'à la " pensée positive». Après avoir dégagé ces manifestations collectives comme signifiantes dans l'ordre historico-conjoncturel, Marcuse s'engage à montrer leur fonction négatrice par rapport à la liberté et à la réalisation de l'homme.

Le jugement marcusien implique une conception discriminante de la liberté, idéellement posée mais cependant objectivement réalisable par une émancipation de l'ordre concret. D'ailleurs, l'idée de liberté comme toute autre n'acquiert sa vérité que lorsque sanctionnée par l'action réelle. Marcuse prétend que l'histoire socio-politique actuelle présente les conditions, objectives et subjectives, nécessaires à l'incarnation de la liberté. Cette affirmation se confirme par la connaissance de la nature de l'utopie.

Mais, avant sa volonté d'imaginer une rationalité émancipatrice, le projet marcusien reste préoccupé par la dimension critique. C'est d'elle, et de la pensée dialectique qui y est corrélative, que découle la conscience claire de l'idéologie.

Sans elle, une compréhension de l'absence de la liberté dans les sociétés industrielles avancées apparaît difficile.

6. Philosophie et révolution, Denoël, Paris, 1969, p. 150. 


\section{L'ABSENCE DE LIBERTÉ DANS LES SOCIETES INDUSTRIELLES AVANCEES}

L'affirmation marcusienne de l'inexistence de la liberté doit être entendue d'une double manière, complémentaire, positive et négative : l'homme des sociétés industrielles avancées connaît une liberté mystifiée et «malheureuse» et il n'existe pas de liberté réelle dans l'ordre socio-politique établi.

Marcuse confirme l'ambiguïté de la liberté dans sa dimension historique en écrivant : «Si la liberté est la faculté de décider de sa propre vie sans priver les autres de cette faculté, alors la liberté n'a jamais été une réalité historique - jusqu'à ce jour» 7 .

Pour appuyer cette thèse, le philosophe américain pratique un recours aux catégories marxiennes et freudiennes soit en les empruntant, soit en s'en inspirant.

Il est indéniable que la pensée marcusienne partage avec Marx la définition de l'idéologie et le constat de son existence dans le processus de production lui-même.

L'idéologie, entendue comme représentation occultée de l'ordre réel, existe par la nature même des conditions de travail dans lesquelles sont jetés les producteurs. Un être aliéné sécrète son propre esclavage qu'il soit social ou politique. La conscience de l'idéologie est d'ailleurs davantage obscure aujourd'hui parce que le processus de production la draine encore plus subtilement. "Que la réalité ait absorbé l'idéologie ne signifie pas cependant qu'il n'y a plus d'idéologie. Dans un sens, au contraire, la culture industrielle avancée est plus idéologique que celle qui l'a précédée ${ }^{8}$.

C'est justement parce que les sociétés industrielles avancées présentent un visage différent des structures capitalistes interrogées par Marx au XIX ${ }^{\mathrm{e}}$ siècle que Marcuse verra la nécessité de se démarquer du père spirituel pour mieux le compléter.

Levons immédiatement les ambiguïtés. Marcuse veut « restaurer la théorie marxiste (et non la) réviser» ${ }^{9}$. C'est donc dire

7. Cité dans A. Vergez, Marcuse, p. 96.

8. L'homme unidimensionnel, p. 36. C'est nous qui soulignons.

9. Contre-révolution et révolte, p. 47. 
qu'il en comprend toute la portée et veut l'actualiser mais n'entend pas en démolir les fondements. Entre Marx et Marcuse, il y a plus une différence de degré que de statut.

Il y a d'abord un fait brut qui s'impose: les altérations successives du capitalisme rendent la critique de Marx incomplète. Le passage du capitalisme concurrentiel au capitalisme actuel est significatif à plus d'un titre. Le capitalisme libéral du XIX $\mathrm{X}^{\mathrm{e}}$ siècle risque de s'effondrer par la révolution. Grèves, manifestations et pamphlets en déchirent les fibres. L'anarchie du système économique concurrentiel entraîne des dysfonctionnements tels qu'à la fin du siècle précédent, l'État intervient dans les rapports de production comme force régulatrice. L'État cesse alors d'être surtout une structure administrative pour avoir une fonction juridique. C'est lui qui, en fixant les règles du marché (isolationnisme, protectionnisme...) détermine la répartition du pouvoir. Le capitalisme monopolistique d'État légitime cet exercice, qui privilégie des unités économiques spécifiques, au nom de l'intérêt national. L'exercice étatique monopolistique sanctionne la dynamique du capital bancaire qui s'assure de la rentabilité de ses investissements en se fusionnant au capital industriel. Ainsi, dans certaines unités économiques, la concurrence est endiguée. Mais comment l'État peut-il intervenir ainsi sans qu'il y ait opposition de la population? L'intervention peut se faire seulement s'il y a dépolitisation, laquelle sera assurée par la science et la technique, nouvelles forces idéologiques.

Le capitalisme des sociétés industrielles avancées renvoie donc à quatre caractères: il est monopolistique, impérialiste, tributaire des contributions de l'État et encore plus lié que le précédent au complexe scientifico-technologique.

Mais là ne sont pas tous les changements au niveau de l'infrastructure. L'homme des sociétés industrielles avancées accepte sa condition: il participe à la cohésion sociale moyennant des gratifications économiques compensatrices. Plus simplement, la structure de consommation s'est substantiellement modifiée de telle façon que le bien-être collectif estompe toute volonté de dépassement de l'ordre social dominant.

De plus, dans les sociétés industrielles avancées, les hommes acceptent la répression comme propriété immanente au sujet, 
nécessaire à l'activité économique et liée à des intérêts politiques préférables à ceux des ennemis quels qu'ils soient.

Ce sont tout autant le processus de production, la société de consommation que «l'équilibre de la terreur» qui obligent à penser différemment la formation idéologique sous le capitalisme.

La lecture de Freud révèle à Marcuse que l'idéologie renvoie pour être comprise à la fois à des fondements sociaux et à l'imaginaire privé. En ce sens, l'analyse marcusienne consolide les thèses marxiennes par le recours freudien. Il y a une "personnalité de l'idéologie» ${ }^{10}$ que Freud n'avait peut-être pas saisie mais qu'il faut mettre à jour. Ces premières lignes de l'ouvrage Éros et Civilisation montre la contribution freudienne et l'institution implicite du couple théorique Marx-Freud:

«Les frontières traditionnelles de la psychologie et de la philosophie sociale et politique sont devenues caduques à cause de la condition de l'homme à l'époque actuelle: les processus psychiques qui furent autrefois autonomes et privés sont en train d'être absorbés par le rôle de l'individu dans l'État, par son existence publique». ${ }^{11}$

Finalement, Marcuse se préoccupe davantage que Marx des instances de rationalisation ou des appareils sociaux par lesquels l'idéologie se canalisera. Marx en avait signifié l'importance. Marcuse le confirmera sans vergogne.

Les possibilités de la pensée marcusienne étant connues, il est désormais possible de répondre à cette interrogation importante: Quels sont les fondements de l'idéologie?

Marcuse convainc de la double présence de fondements objectifs et subjectifs de l'idéologie.

L'analyse subséquente montrera la difficulté de penser le clivage entre les, fondements objectifs et subjectifs. Toutefois, celui-ci a avant tout une fonction méthodologique et didactique.

Cette réserve étant faite, quelles forces sociales contribuent à l'édification de l'idéologie et corrélativement à l'intégration croissante des masses?

10. Éros et Civilisation, p. 233.

11. Idem, p. 9. 
Les sociétés industrielles avancées de type capitaliste se construisent et se légitiment grâce à une double rationalisation: l'une s'opère par le bas dans le processus de production lui-même et l'autre s'exerce par le haut à travers des instances collectives diverses.

Ce mode de production garantit sa perpétuation et sa reproduction par l'institution d'un rapport de réciprocité entre le travailleur et le propriétaire privé ou l'emprise monopolistique. Le travailleur accepte de convertir sa force de travail en valeur d'échange dans la mesure où est repensée la composition organique du capital ${ }^{12}$. Au XIX ${ }^{\mathrm{e}}$ siècle plus particulièrement, celle-ci se traduisait par le capital rattaché aux machines-outils recevant le qualificatif de constant, par le capital variable constitué de la somme d'argent strictement nécessaire pour que l'ouvrier reproduise sa force de travail, par la consommation de la classe socio-économique dominante et par le résidu de surproduction. Dans les sociétés industrielles avancées, l'augmentation de la plus-value n'est plus relative exclusivement à la force productive immédiate qu'est l'ouvrier mais, entre autres, au développement du complexe scientifico-technologique et/de la structure de consommation: l'ouvrier des sociétés industrielles avancées consent à participer au système de production dóminant par la vente de sa force de travail en échange de son intégration à la structure de consommation. L'action du travailleur manifeste, dira Marcuse, son «embourgeoisement». Ainsi, il cautionne l'ordre existant, celui-ci devenant désormais légitimé par la base plutôt que strictement imposé par le haut grâce aux appareils idéologiques et répressifs. La légitimation de la domination se trouve ainsi dans le processus de production lui-même. La structure dominante ne se fonde plus d'abord, en première instance, sur des nécessités naturelles et divines comme au Moyen-Âge ou sur des vérités positives comme à la Renaissance. Il reste cependant que tout un univers de discours doit être créé pour assurer toute l'efficacité de l'idéologie.

La présence de l'idéologie dans le processus de production est reconnue en ces termes:

12. MARX, Karl, Le Capital, Livre 1, Garnier-Flammarion, Paris, 1969, pp. 154-162. 
«L'idéologie d'aujourd'hui réside en ceci que la production et la consommation reproduisent et justifient la domination. Mais leur caractère idéologique ne modifie pas le fait que les profits et les avantages sont réels. La répression de l'ensemble réside dans une large mesure dans son efficacité : elle élève le niveau de culture matérielle, facilite l'obtention des biens de consommation, rend le confort et le luxe meilleur marché, entraîne des secteurs toujours plus vastes dans l'orbite de l'industrie, tout en se faisant en même temps le soutien du labeur de la destruction $» .{ }^{13}$

Mais ce n'est encore là qu'un constat. Ce qu'il importe de savoir c'est pourquoi le processus de production se fait générateur de l'idéologie? Cette interrogation renvoie à la nature même de l'idéologie.

Il y a une dialectique subtile dans le processus de production des sociétés industrielles avancées. La libération garantie tout autant par la société de consommation que par l'appareillage technologique sophistiqué apparaît n'être qu'un simulacre. La libération supposée constitue plutôt une domination renforcée.

La société de consommation a une fonction idéologique en ce qu'elle permet le maintien et la consolidation du pouvoir des classes dirigeantes. La performance de l'idéologie apparaît corrélative au refus de son dépassement et à la proclamation sans ambiguïté du statu quo. Plus l'idéologie est intégrée, plus s'étiole toute opposition. C'est ce que Marcuse nommera «l'engourdissement de la critique».

La fausse conscience créée par la société de consommation s'exprime d'abord par la reconnaissance de "l'égalisation des classes ${ }^{14}$. Elle se manifeste aussi par l'assentiment à l'impérialisme et par l'incompréhension devant la paupérisation de certains groupes sociaux. L'homme des sociétés industrielles avancées accepte que son bien-être soit édifié sur la misère des autres. L'homme des pays du Nord croit que l'ordre réel ne peut être que l'ordre prescrit vis-à-vis de la périphérie. L'idéologie consiste justement à rendre nécessaire ce qui n'est que relatif. S'il consent à la misère éloignée, l'homme des sociétés industrielles avancées lance l'anathème à celle d'ici en rendant coupable celui

13. Eros et Civilisation, p. 99.

14. L'bomme unidimensionnel, p. 33. 
qui la subit plutôt que les structures qui la génèrent. De fait surgit l'un des paradoxes apparents vécus par cet homme: sa «fausse conscience» est toujours bonne.

«La fausse conscience» apparaît encore dans la soumission à la répression conçue par tous comme une nécessité. Le consommateur vit son hédonisme en reconnaissant l'aliénation comme un moyen incontournable et nécessaire pour y parvenir. C'est donc là avoir réduit sa volonté et sa liberté à un acte à vide (avide) pour lequel il est compréhensible de se perdre dans son travail.

Ainsi, le processus d'identification par la consommation de l'homme au pouvoir dirigeant est presque total.

Les sociétés industrielles avancées sécrètent aussi l'idéologie par tout l'appareil scientifico-technologique intégré au processus de production. Cet appareil qui pourrait assurer la libération, notamment par le dépassement de la pénurie, la création de temps de loisir non répressif et/ou la disparition du travail aliéné, sert plutôt à renforcer les formes de répression. Par le mode de rationalité qu'il impose, il crée un type de présence-au-monde qui sanctionne l'ordre dominant. La science et la technique en effet donnent aux hommes des conceptions de soi, des rapports avec la nature et du monde collectif telles que toute activité doit s'incarner dans une fin socialement utile, rentable et opérationnelle. Conséquemment, les hommes des sociétés industrielles avancées se conçoivent comme êtres réifiés. Cette «autoréification» s'exprime par sa participation à des activités socialement utiles, donc s'inscrivant dans l'ordre d'un progrès satisfaisant les intérêts dominants.

La sanction par l'ordre politico-économique de l'appareil scientifico-technologique se comprend mieux désormais. Une raison était claire, l'autre obscure. Bien évidemment, elle se justifie par une conception située du progrès mise en valeur au début du XIX ${ }^{\mathrm{e}}$ siècle et qui depuis s'est raffermie. Mais aussi en structurant le processus de production l'appareil scientificotechnologique façonne la conscience de l'homme et accentue la valeur de l'idéologie par la "glorification de l'adaptation» ${ }^{15}$.

15. Eros et Civilisation, p. 238. 
A ces formes créatrices de l'unidimensionnalité de la pensée s'ajoutent les instances de rationalisation générant l'idéologie par le haut. Ce sont des médiateurs proprement mobilisateurs de la totalité sociale qui savent produire et affirmer l'idéologie. Les circuits idéologiques apparaissent polymorphes: des formes artistiques, le Droit, la famille, l'institution scolaire ou les "fournisseurs d'information de masse ${ }^{16}$ fabriquent la pensée unidimensionnelle. Ces deux derniers canaux idéologiques endiguent la pensée critique et radicale d'une double façon. Ils peuvent montrer une adhésion complète à l'axiologie dominante et ainsi prescrire des attitudes et des comportements spécifiques. Ou ils peuvent feindre la neutralité et l'impartialité dans la mesure où ils préconisent le pluralisme et présentent les phénomènes dans toute leur objectivité. Marcuse récuse tout autant cette seconde attitude :

«Un changement dans cet état de choses - c'est-à-dire (...) des besoins intellectuels et instinctuels nouveaux - obligerait de rompre avec la neutralité bienveillante qui embrasse Marx et Hitler, Freud et Heidegger, Samuel Beckett et Mary McCarthy ; il exigerait la partialité - une éducation à la partialité contre une tolérance et une objectivité qui n'agissent de toute façon que dans la sphère de l'idéologie et dans les domaines qui ne menacent pas l'ensemble» ${ }^{17}$.

Même s'il a été démontré que «l'engourdissement de la critique » relève d'une rationalisation à double niveau, il reste que des forces sociales ne peuvent faire vivre l'homme des sociétés industrielles avancées dans l'idéologie sans une conversion de l'imaginaire. En un mot, la performance de l'idéologie ne peut s'expliquer uniquement par le contrôle répressif des structures. Il est nécessaire que la psychologie individuelle soit transformée. L'idéologie sait d'ailleurs agir en ce sens. D'où le renforcement de la restriction de la liberté.

La pensée sociale et politique marcusienne se double d'une véritable anthropologie philosophique qui se réclame de la psychologie freudienne. Marcuse ne saurait étudier les zones obscures de l'idéologie s'il ne posait dans toute son acuité la question anthropologique: qu'est l'homme des sociétés industrielles avancées? La psychanalyse constitue selon lui un recours

16. L'bomme unidimensionnel, p. 39.

17. Pour une théorie critique de la société, p. 199. C'est nous qui soulignons. 
nécessaire pour y répondre. En plus de «découvrir» ${ }^{18}$ les catégories universelles de la psyché individuelle, elle a compris l'importance d'établir une relation originaire entre le sujet et la réalité sociale pour dévoiler la nature du développement ontogénique. Freud a en effet montré que, malgré l'universalité des structures psychiques, seule une psycho-sociologie peut sonder l'état de l'individu. L'anthropologie philosophique marcusienne suppose cette psycho-sociologie parce que l'interrogation porte au niveau des déterminants sociaux contributifs de la constitution d'une conscience individuelle particulière. L'homme vivant l'aliénation sociale que constitue l'idéologie voit sa psyché transformée. Les intentions de cette manipulation sont d'ores et déjà connues. Toutefois, le processus ne l'est pas.

Pour bien saisir la nature du psychisme de l'homme des sociétés industrielles avancées analysée à la lumière des thèses freudiennes, il importe de remonter au conflit premier ayant dû exister au niveau phylogénique et se reproduisant ontogénétiquement entre le sujet et la réalité. Nombre de concepts freudiens demandent à être introduits. Ils se préciseront en cours d'exposé.

Quoiqu'il reconnaisse qu'elle se manifeste différemment selon les cultures et les moments historiques, Freud soutient l'existence d'une opposition universelle fondamentale entre l'individu et le réel dans toute son organisation.

Originairement et instinctuellement, le sujet, de par son psychisme basé sur le principe de plaisir, s'oppose à la réalité extérieure contraignante. Le sujet recherche le plaisir en agissant en fonction de ses pulsions instinctives. Le «ça » contrôle le sujet. Très tôt, cependant, le principe de réalité par la médiation du père d'abord, des personnages sociaux ensuite, ou des institutions finalement, s'imposera au sujet, d'où le déplaisir. Le principe de réalité, ayant premièrement une connotation concrète, exige du sujet une adaptation par l'inhibition de ses instincts. C'est le règne de la pénurie primitive (Ananké) qui oblige les chefs de toute communauté à soumettre l'individu à des restrictions économiques et érotiques quand, mu par le principe de plaisir, il

18. Le terme est utilisé par Marcuse lui-même. 
recherche la satisfaction totale. "Phylogénétiquement et ontogénétiquement, au cours du progrès de la civilisation et du développement de l'individu, les traces mnémoniques de l'unité entre liberté et nécessité succombent à la répression sociale» ${ }^{19}$. La restriction du principe de plaisir par la civilisation dont les refus spontanés et répétés de l'individu se traduisent pour lui par un sentiment de culpabilité, par du ressentiment ou par l'ostracisme assure la poursuite des développements phylogénique et ontogénique. Une telle répression altère la figure psychique de l'individu. Animé par une volonté de vivre, l'individu, afin d'éviter le déplaisir relié à son refus des prescriptions socioculturelles, acceptera d'orienter ses désirs en les identifiant à ceux sanctionnés par le pouvoir institué. Progressivement, ainsi, l'individu niera sa sphère de besoins privés en l'apparentant à celle des exigences et des nécessités collectives. Le principe de réalité, d'abord extérieur, s'intériorise donc pour faire partie de la psyché individuelle. En ce sens, aux instances du ça et du moi qui forment originairement l'appareil psychique au point de vue topique s'ajoute le surmoi : ce sont là trois régions particulières jouant un rôle déterminé. Le ça a déjà été défini. Le surmoi constitue en quelque sorte ce juge intérieur qui endigue les pulsions instinctuelles et affirme la morale établie pendant que le moi apparaît comme la structure évaluant et organisant l'action du sujet eu égard à son adaptation à la réalité. Le moi, identifiable à la raison, dicte les altérations nécessaires des plaisirs et des désirs pour garantir leur réalisation sociale. "Il a pour mission d'être le représentant du monde extérieur aux yeux du ça, et pour le grand bien de ce dernier» ${ }^{20}$.

Il y a ainsi dans le passage de l'état naturel à l'état de civilisation une inversion qui apparaît entre le principe de plaisir et le principe de réalité. Celui-là originairement premier est subjugué progressivement sous celui-ci. Le plaisir est reporté et dévié dans des formes sociales. Cette déviation peut se traduire en sublimation des pulsions instinctuelles et érotiques. Elle sera alors positive parce qu'elle permettra des productions sociales supérieures. En un mot, le progrès se montre relatif à la

19. Eros et Civilisation, p. 42.

20. Freud, Sigmund, Psychanalyse, P.U.F., Paris, 1971, p. 161. 
canalisation de l'énergie érotique primitive au nom d'exigences collectives.

L'analyse freudienne reproduit donc un rapport syllogistique assez simple. Toute civilisation se construisant sur la raison, laquelle s'incarnant dans la prédominance du principe de réalité, elle apparaît nécessairement répressive. Dans son effort de prêter une signification socio-historique aux concepts freudiens, Marcuse définit la répression comme le processus par lequel la civilisation impose des restrictions aux instincts en fonction de sa survie et d'un progrès vérifiable.

A la suite de Freud ${ }^{21}$, Marcuse peut reconnaître que le processus d'introjection, grâce auquel l'individu s'adapte aux valeurs dominantes et développe des attitudes conformes, constitue une nécessité de tout ordre civilisé. De ce fait, la liberté totale fut jusqu'à aujourd'hui un leurre. C'est dans cet esprit que Marcuse écrit :

"Toute liberté existant dans le domaine de la conscience développée et dans le monde qu'elle a créé n'est qu'une liberté dérivée, une liberté qui est le fruit d'un compromis, une liberté obtenue aux dépens de la satisfaction intégrale des besoins. Et pour autant que la satisfaction intégrale des besoins constitue le bonheur, la liberté dans la civilisation est par essence l'antagonisme du bonheur : elle implique la modification répressive (sublimation) du bonheur $\$ .22$

Dans les sociétés industrielles avancées, ce conflit universel décrit par Freud prend un sens spécifique. Marcuse s'applique à montrer que le processus d'introjection n'opère plus, comme dans les cultures moins évoluées, pour le progrès de la civilisation mais pour des intérêts arbitraires affirmés comme universellement valables et scientifiquement nécessaires. Pour ce faire, Marcuse crée trois nouveaux concepts en s'inspirant de la terminologie freudienne : ce sont ceux de "principe de rendement», de «surrépression» et de «désublimation répressive».

Bien qu'il reconnaisse, à l'instar de Freud, que la pénurie constitue le déclencheur possible de la répression au niveau du développement de la civilisation, Marcuse refuse de croire qu'elle

21. FReud, Sigmund, Malaise dans la civilisation, P.U.F., Paris, 1974.

22. Éros et Civilisation, p. 29. 
soit un fait brut. Selon lui, elle traduit la domination d'un groupe qui, originairement, l'a imposée par la violence et, plus tard, par l'organisation rationnelle du pouvoir. D'où la naissance du principe de réalité qui s'est progressivement mué en principe de rendement dans les sociétés plus avancées. Ces sociétés, structurées autour de la «rationalité de la domination» ${ }^{23}$, se basent sur ce principe d'une triple façon. Premièrement, le maintien de l'aliénation du travail implique une disparition du plaisir libidinal tant corporel que spirituel dans l'activité. Fort simplement, la recherche du profit maximal oblige le sujet à produire un travail parcellaire et monotone ne faisant appel ni à ses facultés intellectuelles ni à sa dextérité grâce auxquelles il se réaliserait dans son activité. Deuxièmement, le travail aliéné impose un temps ludique passif. La société de consommation constitue le lieu privilégié du report de l'activité libidinale du travailleur aliéné. Pourtant, elle-même basée sur le rendement, elle reproduit l'aliénation en faisant de l'individu un être passif qui subit la stimulation des produits et qui est contraint aux lois du marché et aux modes. Conséquemment, le principe de rendement s'exprime dans une double réduction, temporelle et spatiale, de la libido. Les activités de travail aliéné et de consommation accaparent la grande totalité du temps pouvant être dévolu à un plaisir réel. Le plaisir privé est toujours identifié à celui de la collectivité ou accepté comme irréalisable réellement. De plus, le plaisir devient lié à une dimension du corps. Le sujet ne le trouve plus dans l'imaginaire et ne le cherche pas dans la mise en ouvre de certaines habiletés. Il le confine désormais à celui procuré par la consommation. L'hédonisme remplace pour ainsi dire la satisfaction libidinale.

Ainsi «l'appareil fait peser ses exigences économiques, sa politique de défense et d'expansion sur le temps de travail et sur le temps libre, dans le domaine de la culture matérielle et intellectuelle» ${ }^{24}$. Le principe de rendement en remplaçant le principe de réalité n'en réalise pas moins la même fonction: la négation du principe de plaisir.

23. Idem, p. 45.

24. L'bomme unidimensionnel, pp. 28-29. 
Parce qu'elles s'articulent autour du principe de rendement, les sociétés industrielles avancées peuvent être qualifiées de «surrépressives». Dans les sociétés analysées par Freud, la répression constitue un processus nécessaire et positif. Rattachée au développement phylogénétique, la répression apparaît en quelque sorte inconsciente et automatique. La sur-répression s'en distingue en ce qu'elle « résulte de conditions sociales spécifiques et (s'exerce) dans l'intérêt spécifique de la domination» ${ }^{25}$. La sur-répression signifie, plus concrètement, la poursuite de la domination par les appareils idéologiques divers quand les conditions sociales objectives pourraient pourtant garantir la libération réelle du plus grand nombre. Elle ressort donc des contrôles additionnels et renforcés naissant avec les institutions dominantes. Les sociétés industrielles avancées se bâtissent sur ces paradoxes: moins l'aliénation est nécessaire, plus les moyens se raffinent pour la produire; plus l'homme peut être libre, moins il l'est.

Ainsi, une dialectique s'instaure entre le principe de rendement et la sur-répression. Celle-ci génère au niveau de la psyché individuelle celui-là tout autant que le premier produit comme une nécessité le second.

La sur-répression s'exerce d'une façon particulière par la «désublimation répressive ». Selon Freud, le sujet peut orienter et canaliser toute sa libido réprimée dans des formes sociales supérieures qui marquent le progrès de la civilisation. D'après Marcuse, les sociétés industrielles avancées garantissent la «libération de la sexualité dans des modes et sous des formes qui diminuent et affaiblissent l'énergie érotique ${ }^{26}$. L'individu fait dévier, inconsciemment bien sûr, ses désirs dans des circuits qui reproduisent et consolident l'ordre dominant, donc qui bloquent l'exercice de ses pulsions instinctuelles et intensifient sa propre aliénation. Non seulement la libido apparaît-elle réduite spatiotemporellement mais elle ne saurait se manifester en aucune façon dans des formes créatrices. Nombre de manifestations culturelles réfèrent à la « désublimation répressive » en donnant à la sexualité une valeur marchande qui mobilise l'imaginaire et le comportement de l'individu.

25. Eros et Civilisation, p. 88.

26. Idem, p. 12. 
La « désublimation répressive» se situe bien à l’opposé de la sublimation. Parce que permettant un investissement social supérieur, la sublimation se traduit par l'extension de la sexualité et exprime la différence des sujets. Au contraire, la désublimation, tout en affaiblissant l'énergie érotique, l'homogénéise du fait que les hommes des sociétés industrielles avancées l'expriment dans des formes analogues.

Il devient évident que le pouvoir dominant des sociétés industrielles avancées s'exerce grâce à une modification de la structure psychique des individus. S'il y a conflit à l'origine comme dans toute civilisation, il est résolu par la dynamique spécifique des pressions sociales caractéristiques des sociétés industrielles avancées.

L'individu, soumis à la sur-répression, acceptant le principe de rendement et agissant selon la désublimation, constitue un «idéologisé » ${ }^{27}$. Comment se caractérise-t-il en ce qui concerne sa psyché privée?

L'idéologisé se caractérise par une standardisation de ses instances psychiques. Son ça s'uniformise; son surmoi s'identifie à celui de toute la collectivité; le moi emprunte des comportements stéréotypés. "La conscience (...) tend à se limiter à la tâche de régler la coordination de l'individu avec la société ${ }^{28}$.

La standardisation signifie la création d'une seconde nature chez l'idéologisé par une introjection de catégories spécifiques. L'individu introjecte la moralité sociale avec ses exigences, ses interdits et ses dogmes. Celle-ci s'intègre à sa conscience de telle sorte que ses propres choix ne relèvent plus que d'elle. Pourtant, l'individu croit encore à son autonomie quand il assume de fait une morale hétéronome et agit selon les normes de celle-ci. Selon Marcuse, la constitution des masses dans les sociétés industrielles avancées se rapproche des traits régressifs présents dans la horde primitive et identifiés par Freud. Ce dernier les rattache à une nécessité vitale tandis que Marcuse les voit comme les manifestations du processus psychologique de constitution de l'idéologie. La domination de la morale hétéronome s'exprime

27. Ce barbarisme est présent dans Dollê, Jean-Paul, Le désir de révolution, 10/18, Paris, 1975. Il nous apparaît fort pertinent dans ce contexte.

28. Éros et Civilisation, pp. 101-102. 
chez l'idéologisé par un effacement de sa personnalité individuelle consciente, une orientation de ses pensées et de ses sentiments dans des directions similaires à celle de la collectivité, une prépondérance de son affectivité et de la vie psychique inconsciente et une propension à exécuter immédiatement les intentions qui se manifestent.

C'est donc de tout un processus de socialisation qu'il s'agit parce qu'il vise à intégrer l'individu en supprimant son moi privé et en l'identifiant à l'idéal du groupe. Dans les sociétés primitives, la socialisation s'exerce par les membres du groupe immédiat sous l'égide du chef. Elle est davantage médiatisée dans les sociétés industrielles avancées que Marcuse appelle «société sans père». Il s'y trouve une incarnation symbolique du père primitif dominateur dans la division sociale du travail, dans la bureaucratisation, dans l'administration des lois ou dans la consommation de masse. Subtilement « l'autorité sociale est absorbée et travaille comme si elle était son propre désir, sa propre morale et sa propre personnalité» ${ }^{29}$.

Conséquemment, que devient la liberté dans les sociétés industrielles avancées?

Être libre, c'est participer à l'axiologie collective imposée par la nécessité de la répression. Lorsque l'individu agit selon les impératifs de l'ordre établi et qu'il choisit sans jamais interroger ni réviser les fondements et les valeurs, il se prétend libre. Que la conservation de l'ordre existant soit conditionnelle à la réalisation de la liberté est fondamentale. Elle traduit bien l'emprise idéologique. Toute objection contre l'ordre social dominant risque fortement d'être une négation de la liberté. Ainsi, la conscience vit dans un monde concentrationnaire où le possible n'est que ce qui existe.

Le jugement marcusien marque encore une fois par sa sévérité : tout choix, formulé par le sujet, qui réaffirme les seules valeurs de l'ordre existant est sur-répressif, renvoie à un faux besoin et ignore la réalité de la liberté véritable.

Marcuse soutient que la liberté est irréductible à l'exercice d'un choix entre des contraires. La liberté de l'homme des sociétés

29. Idem, p. 53 . 
industrielles avancées reste certes essentiellement une liberté d'adhésion. La liberté réelle se trouve à la fois dans la qualité des contraires et dans le contenu du choix effectué par le sujet: «la liberté humaine ne se mesure pas selon le choix qui est offert à l'individu, le seul facteur décisif pour la déterminer c'est ce que peut choisir l'individu» 30 .

La position marcusienne suppose une possible catégorisation des vrais et des faux besoins et une norme de détermination des contenus non-répressifs. Marcuse s'est confronté à ce double problème.

Le caractère de vérité ou de fausseté d'un besoin résulte d'une réponse donnée suite à la participation à une "méthode rationnelle de libération» ${ }^{31}$ : celle des essais et des erreurs. Marcuse croit que les sujets, placés face à des choix, apprendront, grâce à l'éducation, à discriminer ceux qui sont sur-répressifs et ceux qui sont non-répressifs. La désignation du qualificatif «non-répressif» peut-être prêtée au contenu d'un choix quand il se fonde sur l'impératif catégorique du respect de l'homme ${ }^{32}$. Toute activité, rationnelle ou pratique, qui s'exprime par une limitation des droits de l'homme devient non-libre. Ainsi, parce que par leurs choix économiques, politiques et sociaux et malgré les virtualités de l'appareillage scientifico-technologique, les pays industriels avancés maintiennent le règne de la nécessité qui contraint des masses à l'indigence, à l'aliénation et à l'encerclement de l'imaginaire, ils se font sur-répressifs.

Marcuse parlera donc de la liberté des hommes des sociétés industrielles avancées comme d'une «liberté malheureuse».

Les trois fonctions essentielles de l'idéologie apparaissent maintenant dans toute leur acuité : la mystification, la conservation et la reproduction de l'ordre dominant. Par le processus de production, grâce aux instances sociales diverses et par un «contrôle qui plonge jusqu'au niveau instinctuel et physiologique de l'individu» ${ }^{33}$, l'idéologie réalise ses fonctions. La liberté réelle

30. L'bomme unidimensionnel, p. 33.

31. Éros et Civilisation, p. 207.

32. Idem, p. 187.

33. Contre-révolution et révolte, p. 85. 
reste cependant une possibilité historique. Lorsque la philosophie aura "démontré que la non-liberté est établie au cœur des choses» ${ }^{34}$, elle favorisera le "Grand Refus» ${ }^{35}$ parce qu'elle se pense comme rationalité émancipatrice.

\section{CONDITIONS DE LA LIBERTÉ}

L'incarnation de la liberté découle de l'exigence de la libération manifesté par la volonté de transformation de la rationalité, de la domination et de la conscience de la vérité. "La réalité vraie présuppose la liberté et liberté présuppose la connaissance de la vérité» ${ }^{36}$. En d'autres termes, seules une révélation de la nécessité de la transcendance de l'ordre présent et une édification de l'être de la nouvelle réalité assureront l'instauration de la liberté.

Cette dialectique de la vérité, de la réalité et de la liberté posée par Marcuse débouche sur une triple interrogation: (1) comment instaurer la vérité ? (2) quelle est «la marque de la liberté réalisée» ${ }^{37}$ ? et (3) quel est le profil de la réalité émergeant de la rationalité émancipatrice?

L'affirmation historique de la vérité s'insère dans une compréhension des conditions objectives et subjectives de la situation politique et culturelle. Pour Marcuse, la coprésence d'un prolétariat, d'une couche démographique de marginaux, d'une intelligentzia et d'appareils idéologiques, que ce soit la science, l'art ou l'école, rend probable la transcendance des forces politicoéconomiques des pays industriels avancés. Toutefois, contrairement aux conclusions marxiennes et marxistes, la négation, pour Marcuse, subit un déplacement stratégique. Le prolétariat des sociétés industrielles avancées se distingue de celui du capitalisme concurrentiel du XIX $\mathrm{X}^{\mathrm{e}}$ siècle par son embourgeoisement manifeste, c'est-à-dire par la participation aux intérêts dominants et par la souscription aux contradictions du système. De ce fait, il refuse et cesse d'être la forme déterminante de l'enclenchement

34. Raison et Révolution, p. 44.

35. Marcuse emprunte cette expression à Whitehead.

36. Raison et Révolution, p. 199.

37. F. NiETzSCHE, de mémoire. 
révolutionnaire ${ }^{38}$. La négation opérant le changement qualitatif démarrera et trouvera son impulsion non de l'intérieur mais de l'extérieur, chez ceux-là mêmes qui connaissent l'exclusion et qui s'objectent à toute intégration. L'extrait suivant montre sans équivoque sa position :

«La puissance du négatif naît en dehors de cette totalité répressive, animée par des forces et des mouvements qui n'intègrent pas encore la productivité agressive et répressive de la société dite "d'abondance" ou qui se sont déjà libérés de ce développement, et ont ainsi la chance historique de s'engager dans l'industrialisation et la modernisation en suivant une voie radicalement différente» ${ }^{39}$.

Les forces négatrices subjectives, en tant qu'orientées vers la constitution d'une pensée radicale et d'une civilisation nonrépressive, doivent consolider la valeur de leur projet émancipateur par l'engagement de puissances objectives.

Les mouvements non encore intégrés («outsiders») favoriseront la négation en développant des modèles contre-culturels incitatifs d'une nouvelle sensibilité pour la masse prolétarienne. La nouvelle sensibilité constitue le gage de la suppression progressive des entraves imposées aux besoins instinctuels. D'autant plus que la science et l'art confirment et structurent le dépassement des formes existantes. L'art permet d'imaginer des projets sociaux scientifiquement réalisables et découlant des exigences de la sensibilité personnelle. En ce sens, en s'immisçant dans le politique ${ }^{40}$, l'art s'institue comme médiateur entre le savoir objectif qui détermine les possibilités matérielles concrètes et les besoins sensibles. "Il faudrait (dorénavant), selon Marcuse, chercher la liberté dans la libération de la sensibilité plutôt que dans celle de la raison, et dans la limitation des facultés "supérieures» en faveur des facultés "inférieures» ${ }^{41}$. La libération de la sensibilité ne signifie cependant pas l'expression

38. L'extrait suivant marque bien le refus de Marcuse de penser qu'une altération des sociétés industrielles avancées par voie démocratique garantirait la liberté : « travailler à l'amélioration de la démocratie existante revient manifestement à reporter indéfiniment la date où pourra enfin apparaître une société libre » : Vers la libération, p. 121.

39. "Sur le concept de négation dans la dialectique», pp. 218-219.

40. Dans Vers la libération, p. 72, Marcuse parle de «l'ingérence de l'esthétique dans le politique».

41. Eros et Civilisation, p. 177. 
incontinente de toutes les formes instinctuelles. Marcuse pense que l'ordre de la civilisation non-répressive - qu'il nomme aussi esthétique - ressortira de la tension entre la désublimation de la raison par laquelle tout travail trouvera sa valeur non dans sa fonctionnalité sociale mais parce que répondant à un but personnel et dans l'auto-sublimation de la sensibilité par laquelle le sujet acceptera l'expression de ses désirs dans les limites d'une réalisation commune.

La vérité de l'ordre esthétique découle de la confluence des facteurs subjectifs et objectifs. Une intelligentzia non conformiste, consciente de l'entrée de l'ordre économique dans une phase de "crise structurelle», renforce auprès des producteurs immédiats et des groupes d'exclus l'impératif catégorique du changement, objectivement réalisable parce que non contredit par les lois constatables.

Donc, l'expression réelle de la liberté signifie la création d'un nouveau principe de réalité. La liberté ne saurait se réduire au droit de choisir mais est sociale en ce qu'elle ressort d'un vécu collectif où les sujets individuels acceptent de déterminer objectivement l'ordre public en l'organisant rationnellement. Encore une fois, Marcuse rejoint Marx ${ }^{42}$.

$\mathrm{Si}$, pour Nietzsche, « la marque de la liberté réalisée » est « de ne plus rougir de soi», pour Marcuse, elle se situe plutôt dans la souscription de la conscience autonome à une transmutation du profil éthico-social ${ }^{43}$. Dès qu'une telle souscription devient une nécessité biologique qui s'exprime par «l'incapacité physique de supporter une répression qui ne serait pas indispensable à la protection et à l'amélioration de l'existence » ${ }^{44}$, le sujet peut être dit libre. La nouvelle sensibilité engage désormais à un investissement axiologique inédit dans les relations avec la nature, avec autrui et avec soi-même. L'environnement humain doit être réaménagé pour le démaquiller de toutes les obligations du

42. "Autorité et famille», texte sur Marx, pp. 122-145.

43. Vers la libération, p. 54: "Cette grande révolte anti-autoritaire proclame qu'une société socialiste peut et doit être jolie, claire et gaie, et qu'en l'absence de ces qualités, il est en vain de parler de liberté : elle affirme sa foi en la rationalité de l'imagination, et revendique une autre rationalité et une autre culture».

44. Nous paraphrasons un extrait de Vers la liberation, pp. 58-59. 
principe de rendement et les institutions doivent obtempérer à l'Éros qui connut le refoulement. L'homme libre définit en toute conscience ses choix, refuse de voir objectiver son corps dans un travail aliénant ou dans une consommation consolante ${ }^{45}$, conçoit la sexualité comme non exclusive et détachée des formes socialement acceptées (liens maritaux et monogamie) ${ }^{46}$, participe à la construction d'un langage autre ${ }^{47}$ et réclame une " pacification de l'existence ${ }^{48}$.

Le texte marcusien reste parcimonieux dans la description de la civilisation non-répressive. Parce que Marcuse soutient qu'elle apparaîtra non pas suite à la création de plans aprioristes qui bousculent les volontés personnelles mais, grâce à la méthode des essais et des erreurs, il évite d'en tracer clairement les zones limitrophes. Cependant, il est possible d'affirmer que Marcuse identifie son projet philosophico-politique au socialisme. Maintes fois, il réitère les volontés inhérentes à la pensée socialiste: propriété collective, rapports organiques entre les besoins privés et les fins publiques, travail fondé sur les fonctions esthétique ${ }^{49}$, ludique et altruiste ${ }^{50}$, planification et contribution des modes de production dans une répartition globale des ressources et/ou libération économique grâce aux performances scientifico-technologiques.

La réalisation du socialisme marcusien reste utopique ou futile, pour les uns, et historiquement engagée, pour les autres.

La dialectique de la liberté dans le texte marcusien se fonde sur une double reconnaissance. D'une part, la liberté est l'a priori

45. Vers la libération, p. 168 : «il n'y a pas de liberté pour l'homme s'il n'est libéré de la domination qu'exerce sur lui la marchandise".

46. Marcuse reconnaît dans Fourier l'un des socialistes les plus éclairés. Il écrit : « Fourier est celui des socialistes utopiques qui est le plus près d'élucider le fait que la liberté dépende de la sublimation non-répressive»: Éros et Civilisation, p. 200.

47. Marcuse parle de la nécessité d'« une révolte linguistique systématique, qui fait éclater le contexte idéologique où les mots sont employés»: Vers la libération, p. 70.

48. L'bomme unidimensionnel, pp. 250 et subs.

49. Le jeune Marx des Manuscrits de 1844, Éd. Sociales, Paris, 1972, avait déjà souligné que le travail, en tant que mode dialectique de naturalisation de l'homme et d'humanisation de la nature, signifie un dévoilement de la chose dans son ordre organique. Marx écrit à la page 64: «l'homme sait produire à la mesure de toute espèce et sait appliquer partout à l'objet sa nature inhérente; l'homme façonne donc ainsi d'après les lois de la beauté ".

50. Vers la libération, pp. 147-169. 
de tout investissement émancipateur et d'autre part, elle en constitue l'objectif ultime.

Si la liberté se définit par la qualité du choix et que celui-ci apparaît valable dès qu'il garantit l'actualisation des désirs privés dans une morale altruiste, au moment où le sujet s'engage dans une stratégie transformatrice de la figure sociale, il se désigne comme conscience libre.

La conscience libre résulte elle-même de déterminants socioéducatifs. C'est pourquoi Marcuse parlera d'une souhaitable « éducation à la partialité ${ }^{51}$ qui saurait discriminer les contenus pertinents dans la définition d'une libération et d'une civilisation non-répressive. La pensée trouvera donc sa pleine liberté dans l'attitude critique et radicale donnée par l'éducation.

L'engagement sécrète son origine dans la liberté. Une liberté qui, en prescrivant la libération, s'auto-engendre et se soumet au critérium de la réalité factuelle et objective. Ainsi, c'est l'instauration d'une rationalité nouvelle qui apparaît fondatrice de la vérité de la liberté.

Tant que la philosophie reste dissociée de la pratique matérielle, son discours conserve un caractère abstrait et idéologique. Le projet marcusien de société libre devient vrai, selon les critères du matérialisme dialectique, lorsque matérialisé historiquement. Marcuse ne quitte pas la topique marxienne:

« La raison a toujours existé, mais pas toujours sous la forme rationnelle. La critique peut donc prendre son point de départ dans n'importe quelle forme de la connaissance théorique ou pratique, et en partant des formes spécifiques de la réalité existante, il peut développer la réalité vraie comme son but et son objectif ultime» 52 .

Marcuse pense donc la liberté dans l'exigence d'une double rationalité, scientifico-technologique et esthétique, mettant fin à l'irrationalité de la modernité.

Département de philosophie

Collège de la Gaspésie

51. "L'individu dans la grande société», p. 199.

52. Lettre de Marx à Ruge, sept. 1843, citée dans H. Lefebvre, Marx, SUP, PUF, Paris, 1972 , p. 86. 


\section{BIBLIOGRAPHIE *}

ASSOUN, P.L. et RAULET, G., Marxisme et théorie critique, PBP, Paris, 1978. MARCUSE, HERBERT, «Sur la philosophie concrète» (1929), in Pbilosophie et Révolution, Denoël, Gonthier, Paris, 1969, pp. 121-156.

"Etude sur l'autorité et la famille» (1936), in Pour une théorie critique de la société, Denoël, Gonthier, Paris, 1971, pp. 9-163.

Raison et Révolution (1939), Minuit, Paris, 1968. Éros et Civilisation (1955), Points, Seuil, Paris, 1970.

Le marxisme soviétique (1958), Idées, Gallimard, Paris, 1963.

, «Note sur la dialectique» (1960), in Raison et Révolution, pp. 39-50.

L'bomme unidimensionnel (1964), Minuit, Paris, 1968.

, «L'individu dans la grande société» (1966), in Pour une théorie critique de la société, pp. 165-207.

, «Sur le concept de négation dans la dialectique» (1966), in Pour une

théorie critique de la société, pp. 209-219.

«La notion de progrès à la lumière de la psychanalyse» (1968), in Eros et Civilisation pp. 251-271.

La fin de l'utopie, Seuil, Paris, 1968.

Vers la libération, Denoël, Gonthier, Paris, 1969.

PALMIER, J.M., Sur Marcuse, 10/18, Paris, 1968.

VERGEZ, André, Marcuse, PUF, Paris, 1970.

* Pour les ouvrages de Marcuse nous indiquons l'année de rédaction entre parentèses. 